\title{
Neuronal Dual Leucine Zipper Kinase Mediates Inflammatory and Nociceptive Responses in Cyclophosphamide-Induced Cystitis
}

\author{
Chen Jiang Weilin Fang Tingting Lv Yinjun Gu Jianwei Lv \\ Renji Hospital, School of Medicine, Shanghai Jiao Tong University, Shanghai, China
}

\section{Keywords}

Dual leucine zipper kinase · Cystitis - Bladder pain ·

Inflammation · Sensory neuron

\begin{abstract}
Interstitial cystitis is associated with neurogenic inflammation and neuropathic bladder pain. Dual leucine zipper kinase (DLK) expressed in sensory neurons is implicated in neuropathic pain. We hypothesized that neuronal DLK is involved in the regulation of inflammation and nociceptive behavior in cystitis. Mice deficient in DLK in sensory neurons (cKO) were generated by crossing DLK floxed mice with mice expressing Cre recombinase under Advillin promoter. Cystitis was induced by cyclophosphamide (CYP) administration in mice. Nociceptive behavior, bladder inflammation, and pathology were assessed following cystitis induction in control and CKO mice. The role of DLK in CYP-induced cystitis was further determined by pharmacological inhibition of DLK with GNE-3511. Deletion of neuronal DLK attenuated CYP-induced pain-like nociceptive behavior and suppressed histamine release from mast cells, neuronal activation in the spinal cord, and bladder pathology. Mice deficient in neuronal DLK also showed reduced inflammation induced by CYP
\end{abstract}

karger@karger.com www.karger.com/jin

Karger"

BOPEN ACCESS
(C) 2021 The Author(s)

Published by S. Karger AG, Basel

This is an Open Access article licensed under the Creative Common Attribution-NonCommercial-4.0 International License (CC BY-NC) (http://www.karger.com/Services/OpenAccessLicense), applicable to the online version of the article only. Usage and distribution for commercial purposes requires written permission. and reduced c-Jun activation in the dorsal root ganglia (DRG). Pharmacological inhibition of DLK with GNE-3511 recapitulated the effects of neuronal DLK depletion in CYP treatment mice. Our study suggests that DLK is a potential target for the treatment of neuropathic pain and bladder pathology associated with cystitis.

(C) 2021 The Author(s)

Published by S. Karger AG, Basel

\section{Introduction}

Interstitial cystitis or painful bladder syndrome is an idiopathic condition of the bladder that has multiple clinical manifestations such as urinary urgency and frequency and discomfort in the pelvic and bladder region. The disease affects approximately 10.6/10,000 of the population with more females affected and adversely impacts patients' daily life [1]. Although the cause of the disease is largely unknown, potential etiologies include neurogenic inflammation and mast cell activation $[2,3]$.

Mast cells play an important role in the pathogenesis of interstitial cystitis with their number elevated in bladder tissue of patients with the disease [4]. These cells are involved in pain regulation by triggering inflammation 
$[5,6]$. Mice deficient in mast cells showed abrogation of bladder pathophysiology and pelvic pain induced by pseudorabies virus [4]. Upon degranulation induced by various stimuli such as damage to the nerve, mast cells release histamine to mediate neuropathic pain [7]. Along with accumulation of mast cells, increased levels of histamine metabolites are detected in the urine of patients with interstitial cystitis [8]. Previous studies have revealed that histamine released from mast cells is implicated in the pain behavior of a mouse model of cystitis and a mouse with disrupted histamine receptor showed reduced pelvic pain [4]. In fact, increased urinary histamine is used as a factor for the diagnosis of interstitial cystitis [9].

Additionally, neuronal activation as indicated by cFos expression and elevated inflammatory responses including neutrophil migration and increased cytokine production are also implicated in cystitis pathogenesis [10]. In the mouse model of cyclophosphamide (CYP)induced cystitis, CYP induced upregulation of c-Fox expression in the spinal cord, increased myeloperoxidase (MPO) activity which is an indication of neutrophil accumulation, and promoted production of proinflammatory cytokines, including interleukin (IL)- $1 ß$ and tumor necrosis factor (TNF)- $\alpha$.

In this study, we aimed to determine the intracellular signaling implicated in the pathophysiology of cystitis and to seek for a potential therapeutic target that may alleviate bladder pain and neurogenic inflammation in cystitis. One such potential signaling molecule is the dual leucine zipper kinase (DLK), also known as mitogen-activated protein kinase 12 (MAP3K12). DLK is a multifunctional protein implicated in a wide range of pathophysiological conditions of neurons including neuropathic pain, axonal degeneration and regeneration, and neuronal survival and death following nerve injury [11, 12]. Pharmacological inhibition and genetic ablation of DLK have been shown to alleviate nerve injury-induced mechanical hypersensitivity [11]. Deletion of DLK in sensory neurons using Wnt1a-Cre-mediated recombination suppresses neuroinflammation by inhibition of cytokine and chemokine production [13].

We hypothesized that DLK in sensory neurons is involved in the pathophysiology of interstitial cystitis and that specific depletion of DLK in sensory neurons may suppress bladder pain and alleviate bladder pathology by suppressing neuroinflammation and neuronal activation. We tested our hypothesis by investigating the effect of DLK conditional deletion in sensory neurons by AdvillinCre (Adv-Cre)-mediated recombination on CYP-in- duced cystitis in mice and explored the potential mechanism underlying DLK-mediated regulation in bladder pain. We also confirmed our findings with pharmacological inhibition of DLK using GNE-3511. GNE-3511 is a specific DLK inhibitor with a low Ki of $0.5 \mathrm{nM}$ that has been shown to exert dramatic protection of neurons from degeneration both in vitro and in vivo [14] and attenuate neurodevelopmental and behavioral defects [15] and neuropathic pain [11], with prospective therapeutic potential in multiple indications.

\section{Materials and Methods}

Mice

A cystitis mouse model was generated by a single intraperitoneal injection of CYP at the dose of $300 \mathrm{mg} / \mathrm{kg}$ in saline according to a previous procedure [10]. Mice were subjected to behavioral assessment at indicated time points and were sacrificed for biochemical and histological analyses at $6 \mathrm{~h}$ following CYP injection.

To specifically delete DLK in sensory neurons, DLK ${ }^{\mathrm{fl} / \mathrm{ll}}$ mice with a background of C57BL/6 J were generated by Biocytogen (Beijing, China) as described previously [13]. Adv-Cre mice (ID: 032536; The Jackson Laboratory) were purchased from The Jackson Laboratory. DLK ${ }^{\mathrm{fl} / \mathrm{fl}}$ female mice were crossed with Adv-Cre mice to obtain Adv-Cre:DLK ${ }^{\mathrm{fl} / \mathrm{fl}}(\mathrm{cKO})$ mice so that Cre-recombinase-mediated recombination is confined to the peripheral sensory neurons [16]. DLK ${ }^{\mathrm{f} / \mathrm{fl}}$ (WT) mice were used as control. For breeding, cKO male mice were crossed with WT female mice.

To determine the effect of DLK inhibition in mice with cystitis, a single dose of DLK inhibitor GNE-3511 (Catalog No. HY-12947; MedChemExpress) was administered at a dose of $75 \mathrm{mg} / \mathrm{kg}$ with $10 \mathrm{mg} / \mathrm{kg}$ of $7.5 \mathrm{mg} / \mathrm{mL}$ GNE-3511 solution by oral gavage at $2 \mathrm{~h}$ prior to CYP injection [11]. Mice were housed in an environmentally controlled facility with a $12 \mathrm{~h} / 12 \mathrm{~h}$ light/dark cycle and free access to water and food. All procedures were approved by the ethics committee of Renji Hospital, School of Medicine, Shanghai Jiao Tong University.

\section{Assessment of Nociceptive Behavior}

Nociceptive behavior and referred hyperalgesia in cystitis mice were assessed according to a previous procedure [17]. Nociceptive behavior was examined at 3.5-4 h after CYP injection. In brief, mice were acclimated on a raised wire mesh for $1 \mathrm{~h}$ prior to observation. Nociceptive behavior was defined as licking of the external urethral opening by the mice. The number of nociceptive behaviors was counted for $30 \mathrm{~min}$. Following the assessment of nociceptive behavior, mice were subjected to the von Frey test to assess referred hyperalgesia. In brief, 4 von Frey filaments with strengths of $0.008,0.07,0.4$, and $1.0 \mathrm{~g}$, respectively, were used sequentially to mechanically stimulate the mice between the urethral opening and the anus. Referred hyperalgesia was scored by the following criteria: a score of 0 indicates that the experimental mouse showed no response at the stimulation; a score of 1 indicates that the mouse bit or licked the area at or around the external urethral opening, bent the trunk, thrashed limbs, raised upper body, and left the position; and a score of 2 indicated that the mouse jumped. Each mouse was stimulated with the same von Frey filament for 10 times, and the total score was recorded. 


\section{Mast Cell Quantification}

Mast cells were visualized through acidified toluidine blue staining as described previously [4]. In brief, bladder tissues were isolated from experimental mice, fixed, embedded in paraffin, sectioned, and stained with acidified toluidine blue. Toluidine-bluepositive cells were counted from 2 sections, and the average number was used for each mouse.

\section{Enzyme-Linked Immunosorbent Assay}

The levels of monocyte chemoattractant protein-1 (MCP-1), IL-1ß, and TNF- $\alpha$ in bladder tissue and histamine in urine were determined by ELISA using relative ELISA kits according to the instructions provided by the manufacturer (R\&D Systems, Minneapolis, MN, USA). For ELISA of MCP-1, IL-1ß, and TNF- $\alpha$, the bladder tissues were isolated from experimental mice and homogenized in lysis buffer provided in the ELISA kit. For ELISA of histamine, urine was collected from experimental mice and diluted in the buffer provided in the ELISA kit.

\section{Immunohistochemistry}

Neuronal activity in the spinal cord was assessed by c-Fos expression as described previously [13]. In brief, $6 \mathrm{~h}$ following CYP administration, mice were perfused under anesthesia and the spinal cord was isolated and postfixed in $4 \%$ paraformaldehyde. After dehydration with $30 \%$ sucrose, L5 spinal cord cryosections were stained with anti-c-Fos antibody (Catalog No. 2250; Cell Signaling Technology) and secondary antibody conjugated to fluorescent dye. c-Fos-stained sections were imaged under a fluorescent microscope, and the number of c-Fos-positive cells in each section was counted.

To determine c-Jun activation in dorsal root ganglion (DRG) neurons, L5 DRGs were dissected out from relevant mice following perfusion. After postfixation, dehydration, and cryosectioning, DRG sections were costained with p-c-Jun (Catalog No. 9146; Cell Signaling Technology) and NeuN (Catalog No. MAB377; MilliporeSigma) primary antibodies and corresponding fluorescent secondary antibodies. DRG sections were then imaged under a fluorescent microscope, and the number of p-c-Jun-positive neurons was counted.

\section{Bladder Edema and Hemorrhage Assessment}

Following indicated treatments in respective mice, the bladder was dissected, weighed, and grossly evaluated in aspects of edema and hemorrhage according to a previous study [10]. In brief, bladder edema was assessed according to the following grading criteria: a score of 3 was assigned to severe edema with fluid detected in the bladder walls; a score of 2 was assigned to moderate edema with fluid detected only in the internal mucosa; a score of 1 was assigned to mild edema with little fluid detected; and a score of 0 was assigned to normal bladder tissue in the absence of edema. Bladder hemorrhage was graded as follows: a score of 0 indicated absence of bleeding, a score of 1 indicated bladder vascular dilatation, a score of 2 indicated presence of mucosal hematomas, and a score of 3 indicated findings of intravesical clots.

\section{MPO Activity Assay}

MPO activity in the bladder was assessed to determine recruitment of neutrophils to the bladder as described previously [10]. In brief, the bladders from the experimental mice were extracted and homogenized. After centrifugation at $4^{\circ} \mathrm{C}$ for $15 \mathrm{~min}$ at $4,000 \mathrm{~g}$, the supernatant was discarded and the homogenate was resuspended with hexadecyltrimethyl ammonium bromide buffer. Following another centrifugation at the same conditions, MPO assay was performed by mixing the supernatant $(25 \mu \mathrm{L}$ per sample) with a solution containing $0.3 \mathrm{~mm}$ hydrogen peroxide, $80 \mathrm{mM} \mathrm{NaPO}_{4}$, and 1.6 $\mathrm{mM}$ tetramethylbenzidine. The optical density was acquired by measuring the absorbance at $595 \mathrm{~nm}$.

\section{Western Blot}

The protein levels of phosphorylated c-Jun and total c-Jun were assessed by Western blot assay as described previously [17]. In brief, L5 DRGs were extracted at $6 \mathrm{~h}$ following CYP treatment and homogenized in radioimmunoprecipitation buffer containing $0.1 \%$ SDS, $0.5 \%$ sodium deoxycholate, and $1 \%$ Igepal CA-630 in phosphatebuffered saline supplemented with protease and phosphatase inhibitors. Proteins were separated in a sodium dodecyl sulfate (SDS)-polyacrylamide gel, transferred onto a polyvinylidene fluoride membrane, and incubated in a primary antibody at $4^{\circ} \mathrm{C}$ overnight. After incubation in corresponding horseradish peroxidase-conjugated secondary antibodies, proteins were shown by an enhanced chemiluminescence detection reagent. Primary antibodies used in this study include anti-p-c-Jun (Catalog No. 9146; Cell Signaling Technology), anti-c-Jun (Catalog No. 9165; Cell Signaling Technology), and anti- $\beta$-actin (Catalog No. ab6276; Abcam). All antibodies were purchased from Abcam (Cambridge, MA, USA).

\section{Statistical Analysis}

Differences between 2 indicated groups were assessed by Student's two-sided $t$ tests or one-way ANOVA analysis with Tukey's post hoc test through GraphPad Prism. The sample size is given in figure legends. Each $n$ represents the number of mice used in the experiment. ${ }^{*} p<0.05,{ }^{* *} p<0.01,{ }^{* * *} p<0.001$, and ${ }^{* * * *} p<0.0001$.

\section{Results}

\section{Deletion of Neuronal DLK Attenuates CYP-Induced Pain-Like Nociceptive Behavior}

To determine the role of neuronal DLK in CYP-induced cystitis in mice, we generated $\mathrm{DLK}^{\mathrm{fl} / \mathrm{fl}}$ mice, specifically ablated DLK in sensory neurons by Advillin-Cremediated recombination [16], and induced cystitis by intraperitoneal injection of CYP. We then assessed nociceptive behavior in these mice and relevant control mice at $3.5 \mathrm{~h}$ following CYP administration. Consistent with the presence of bladder pain, CYP treatment in WT mice substantially increased the number of nociceptive behaviors within the 30 -min observation window compared to vehicle-treated WT mice (Fig. 1a). Vehicle treatment in cKO mice with DLK deletion in sensory neurons did not significantly impact nociceptive behavior compared to vehicle-treated WT mice. Importantly, DLK ablation in sensory neurons significantly reduced the number of nociceptive behaviors compared to that of WT mice following CYP treatment. 
Fig. 1. Deletion of neuronal DLK attenuates pain-like nociceptive behavior. Cystitis was induced by CYP treatment in WT $\left(\mathrm{DLK}^{\mathrm{fl} / \mathrm{fl}}\right)$ and cKO (Adv-Cre:DLK $\left.{ }^{\mathrm{fl} / \mathrm{fl}}\right)$ mice, and the respective control groups for $\mathrm{WT}$ and $\mathrm{cKO}$ mice were treated by vehicle (saline) (a-e). Nociceptive behavior was observed 3.5-4 $\mathrm{h}$ after CYP treatment in $\mathrm{WT}$ and cKO mice (a). Hyperalgesia under different von Frey hair, 0.008 (b), 0.07 (c), $0.4(\mathbf{d})$, and $1.0 \mathrm{~g}(\mathbf{e})$, stimulations was evaluated $4 \mathrm{~h}$ after CYP treatment. $n=10$. Significance was evaluated between WT and cKO mice receiving the same treatment. ${ }^{* *} p<0.01$ and ${ }^{* * *} p<0.001$. Error bars represent SEM. ns, not significant; DLK, dual leucine zipper kinase; CYP, cyclophosphamide.

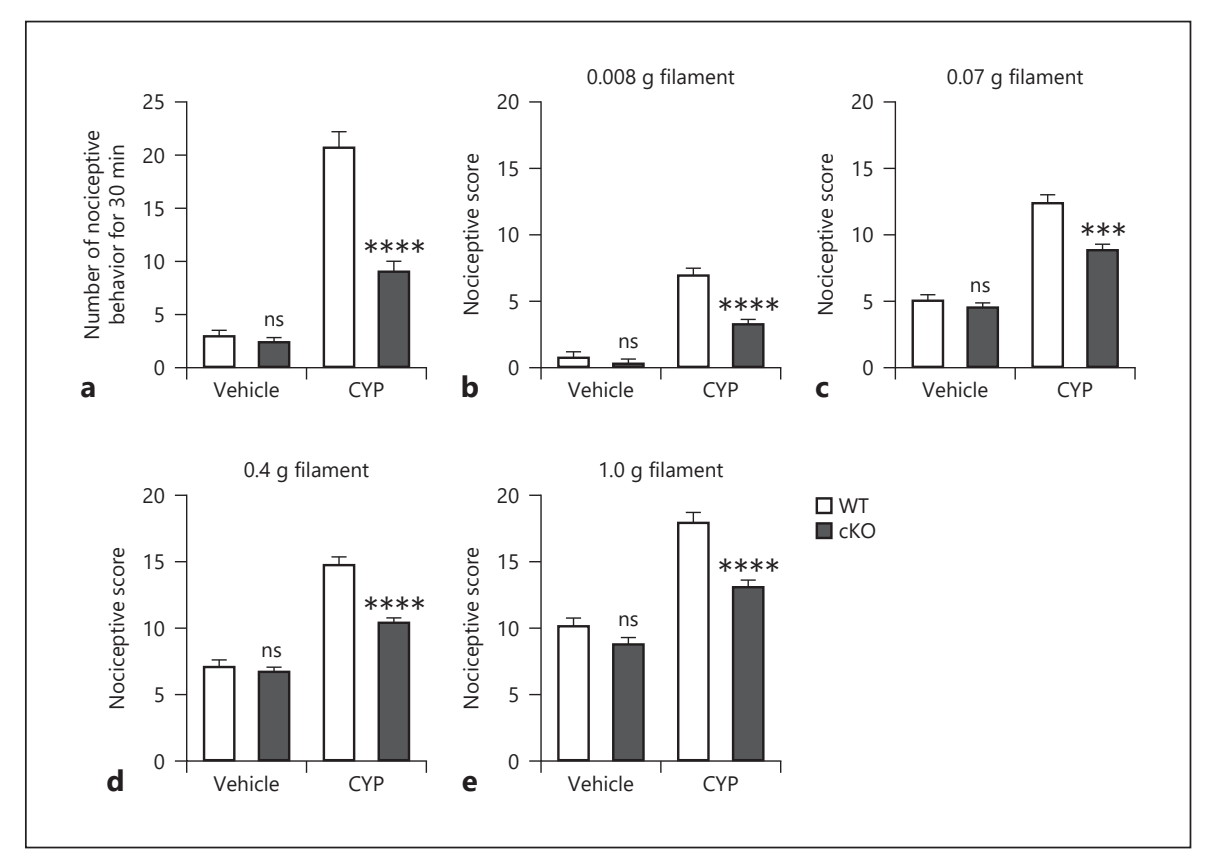

Immediately after assessing nociceptive behavior, mice were subjected to von Frey assay to evaluate referred hyperalgesia. Four von Frey filaments of different strengths were used to stimulate the mice with 10 challenges for each filament. Similarly, we found that CYP administration lead to increased nociceptive score compared to vehicle treatment during all challenges (Fig. 1be) and neuronal ablation of DLK did not induce hyperalgesia in vehicle-treated mice. Consistent with suppression of nociceptive behavior, DLK cKO mice showed significantly reduced nociceptive scores after CYP treatment. Taken together, ablation of DLK in sensory neurons attenuated pain-like nociceptive behavior in the mouse model of CYP-induced cystitis.

\section{Neuronal DLK Modulates Histamine Release and Spinal Neuron Activation in Mice with CYP-Induced Bladder Pain}

We next determined that histopathological mechanism underlying neuronal DLK ablation induced attenuation of pain-like nociceptive behavior in mice treated with CYP. We asked whether neuronal DLK was involved in the regulation of histamine release from mast cells that are important for the pathogenesis of cystitis. To address this possibility, we first examined the level of mast cells in the bladder tissue of DLK cKO mice collected at $6 \mathrm{~h}$ after CYP administration. Quantification of mast cells labeled with toluidine blue showed that CYP significantly elevated the number of mast cells in WT bladder tissue compared to vehicle treatment (Fig. 2a). Vehicle treatment in mice deficient in neuron DLK did not significantly change the level of mast cells compared to that of WT mice. Importantly, deletion of DLK in sensory neurons significantly reduced the number of mast cells in mice treated with CYP.

We then assessed the levels of histamine in the urine of the experimental mice. We found that in WT mice, CYP treatment drastically increased histamine level compared to vehicle treatment. Vehicle treatment in mice with conditional depletion of neuronal DLK did not show significant difference in urine histamine level compared to WT mice (Fig. 2b). On the other hand, mice deficient in neuronal DLK showed significantly reduced histamine in the urine after CYP treatment compared to CYP-treated WT mice.

Additionally, we assessed c-Fos expression in $\mathrm{cKO}$ mice treated with CYP to determine neuronal activation (Fig. 2c). Consistent with a previous finding, CYP treatment significantly increased the number of c-Fos-positive cells in the spinal cord of WT mice (Fig. 2d). We found that neuronal ablation of DLK had no impact on neuronal activation in vehicle-treated mice. However, loss of neuronal DLK significantly suppressed CYP-induced neuronal activation manifested by reduced c-Fospositive cells.

\section{Deletion of Neuronal DLK Suppressed CYP-Induced \\ Edema and Hemorrhage in the Bladder}

We confirmed that CYP treatment in WT mice showed increased bladder weight (Fig. 3b), edema (Fig. 3a, c), and 
Fig. 2. Deletion of neuronal DLK attenuates pain-like nociceptive behavior by modulating the release of histamine and the activation of spinal neuron. Cystitis was induced by CYP treatment on WT and cKO mice (a-d). Bladder tissues from WT and cKO mice were harvested for assay $6 \mathrm{~h}$ after cystitis induction, and mast cells were quantified in paraffin sections following toluidine blue staining (a). The level of urinary histamine was evaluated by ELISA (b). L5 spinal cords from WT and cKO mice were harvested for c-Fos staining $6 \mathrm{~h}$ after CYP treatment (c-d), and the representative images are shown (c), and c-Fospositive cells were counted (d). $n=6$. Significance was evaluated between WT and cKO groups in the same conditions. ${ }^{* *} p<$ 0.01 and ${ }^{* * *} p<0.001$. Error bars represent SEM. ns, not significant; DLK, dual leucine zipper kinase; CYP, cyclophosphamide.

Fig. 3. Deletion of neuronal DLK decreases edema and hemorrhage caused by CYP. Bladder tissues from WT and cKO mice were harvested after cystitis induction ( $\mathbf{a}$ d). a The representative HE images of bladder morphology. The wet weight of empty bladder (b), macroscopic edema (c), and hemorrhage (d) were evaluated. $n=6$. Significance was evaluated between WT and cKO groups in the same conditions. ${ }^{* *} p<$ 0.01 and ${ }^{* * *} p<0.001$. Error bars represent SEM. ns, not significant; DLK, dual leucine zipper kinase; CYP, cyclophosphamide.
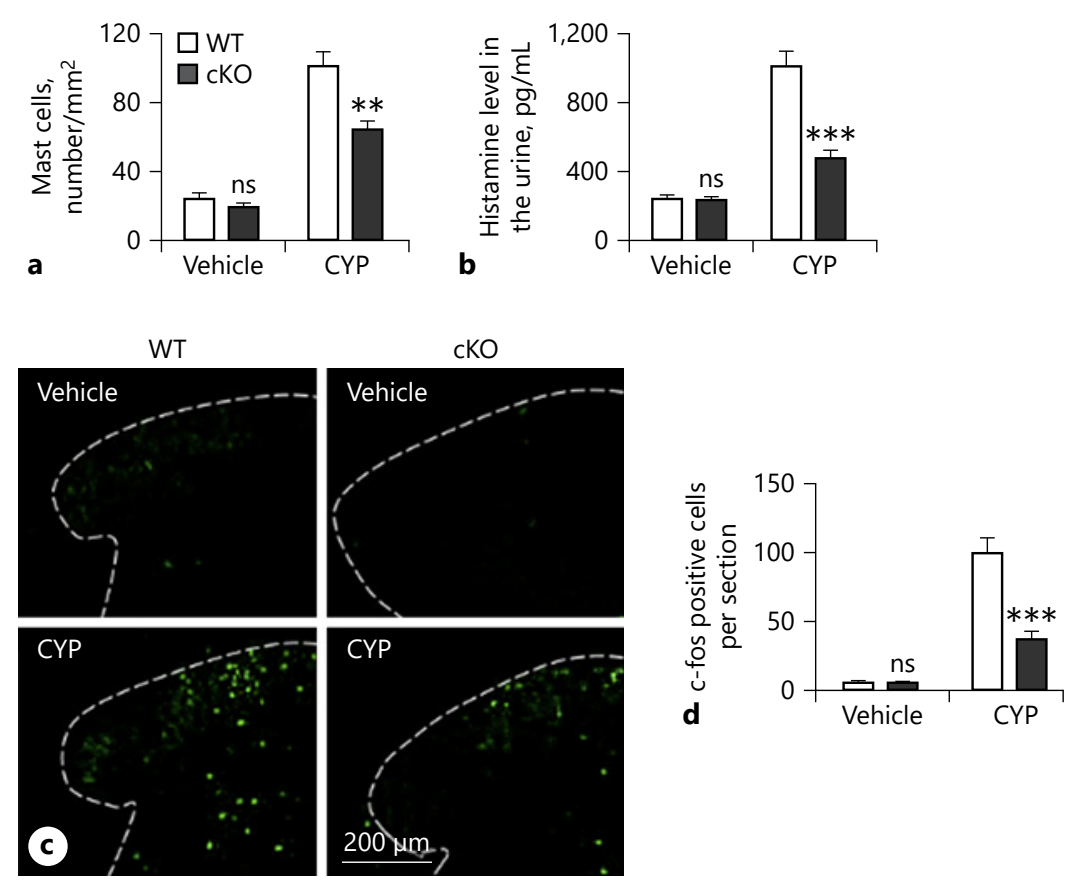

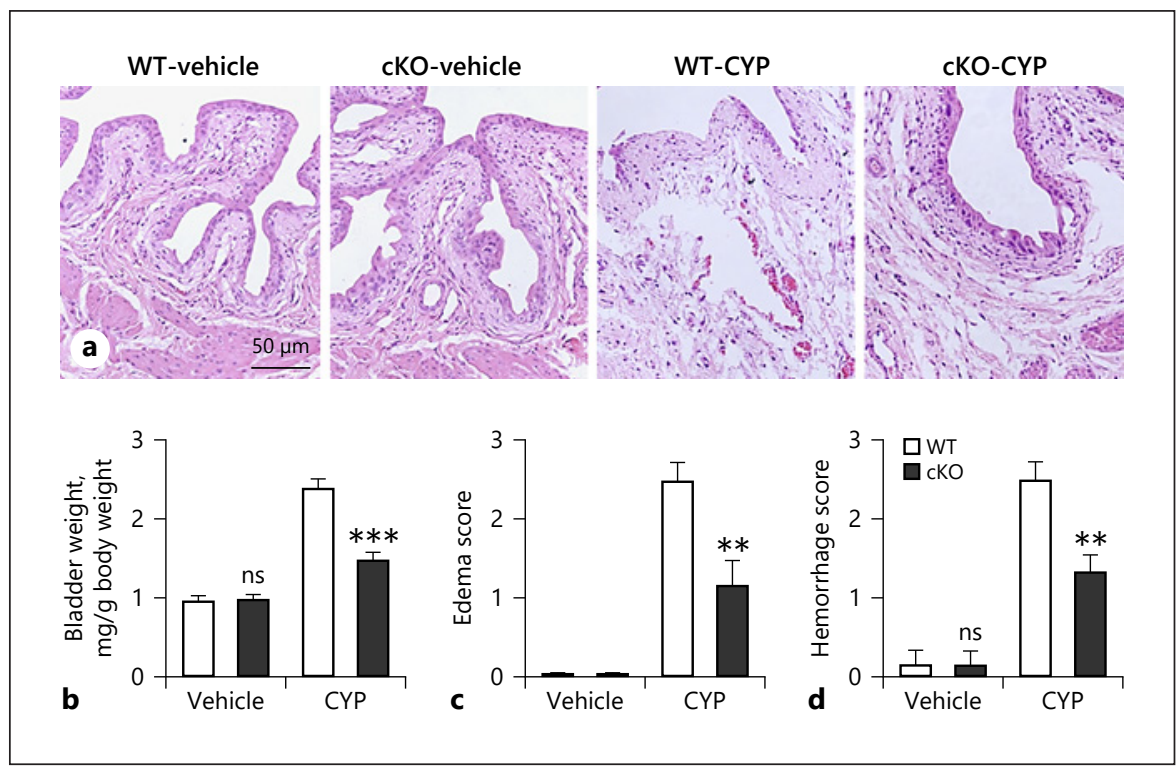

hemorrhage (Fig. 3a, d). Vehicle treatment in mice deficient in neuronal DLK had no impact on the overall bladder morphology and gross assessment. Importantly, deletion of neuronal DLK partially but significantly protected mice from CYP-induced edema and hemorrhage and the bladder weight in CYP-treated cKO mice was also lower than that of CYP-treated WT mice.

Neuronal Dual Leucine Zipper Kinase Mediates Inflammation

\section{Deletion of Neuronal DLK Alleviated CYP-Induced Inflammation}

We assessed bladder tissue inflammation in WT and DLK cKO mice treated with vehicle or CYP. CYP treatment leads to increased MPO activity, an indication of neutrophil migration, in the bladder tissue [10]. Similarly, in WT mice, we also detected an elevation of MPO activity following CYP treatment compared to vehicle- 
Fig. 4. Deletion of neuronal DLK alleviates inflammation caused by CYP. WT and cKO mice were treated by vehicle and CYP, and bladder tissues were harvested $6 \mathrm{~h}$ posttreatment (a-d). MPO activity was determined as described in Materials and Methods (a). The expression level of MCP1 (b), TNF- $a(\mathbf{c})$, and IL-1ß (d) in bladder tissues was determined by ELISA. $n=6$. Significance was evaluated between WT and $\mathrm{cKO}$ groups in the same conditions. ${ }^{*} p<0.05$ and ${ }^{* *} p<0.01$. Error bars represent SEM. ns, not significant; DLK, dual leucine zipper kinase; CYP, cyclophosphamide; MPO, myeloperoxidase; MCP-1, monocyte chemoattractant protein-1; TNF- $\alpha$, tumor necrosis factor- $\alpha$; IL- $1 \beta$, interleukin-1ß.
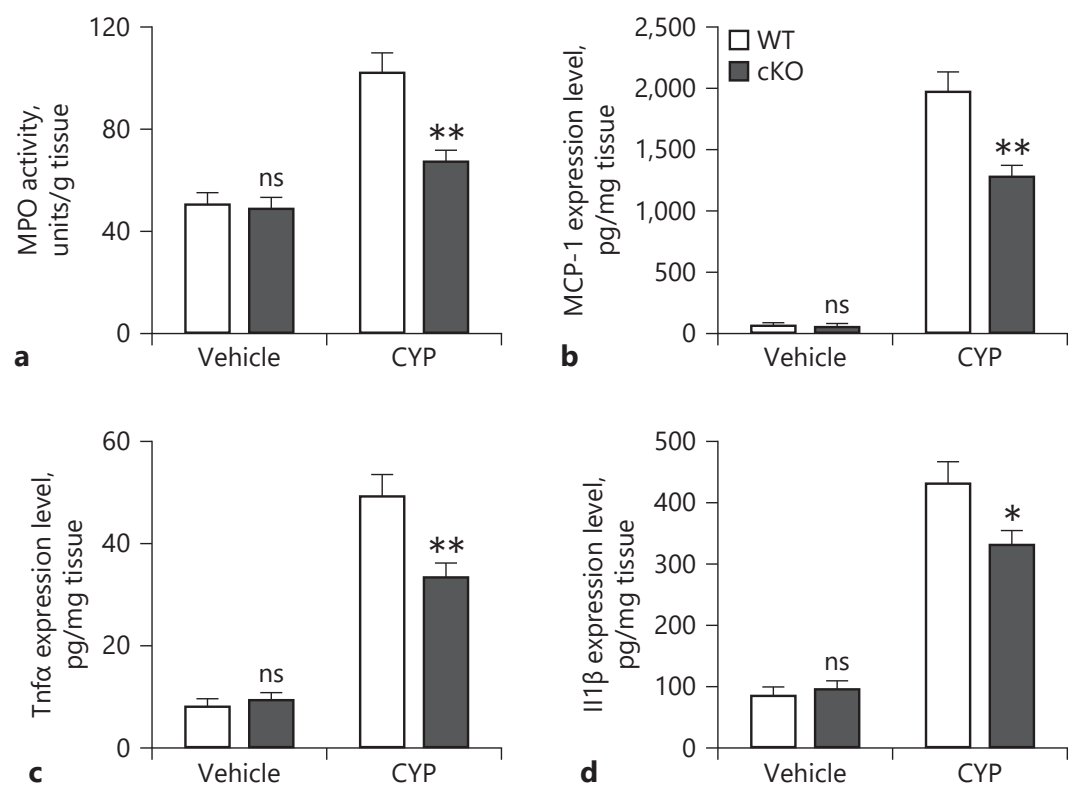

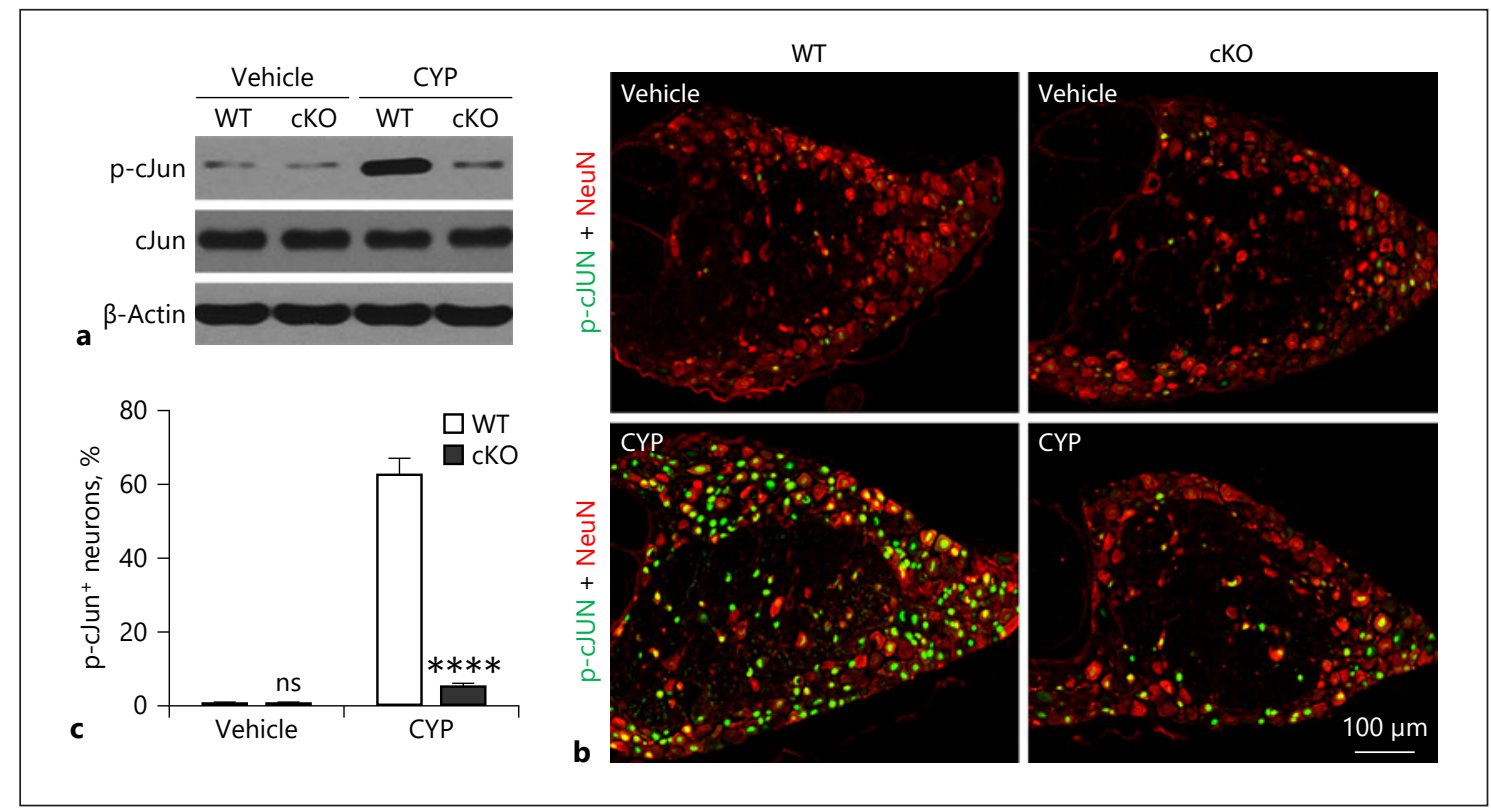

Fig. 5. DLK mediates the activation of c-Jun in DRG upon CYP treatment. WT and cKO mice were treated by vehicle and CYP, and DRGs were harvested $6 \mathrm{~h}$ posttreatment $(\mathbf{a}-\mathbf{c})$. L5 DRGs were harvested for immunoblotting on $\mathrm{p}$-c-Jun, and 1 representative sample from 4 samples is presented (a). $n=4$. Also, L5 DRGs were harvested for immunohistochemical staining on $\mathrm{p}$-c-Jun and $\mathrm{NeuN}$, and 1 representative image is presented $(\mathbf{b})$, and p-c$\mathrm{Jun}^{+}$neurons were counted $(\mathbf{c}) . \mathrm{n}=6$. Significance was evaluated between WT and cKO groups in the same conditions. ${ }^{* * * *} p<$ 0.0001. Error bars represent SEM. ns, not significant; DLK, dual leucine zipper kinase; CYP, cyclophosphamide; DRG, dorsal root ganglia. 


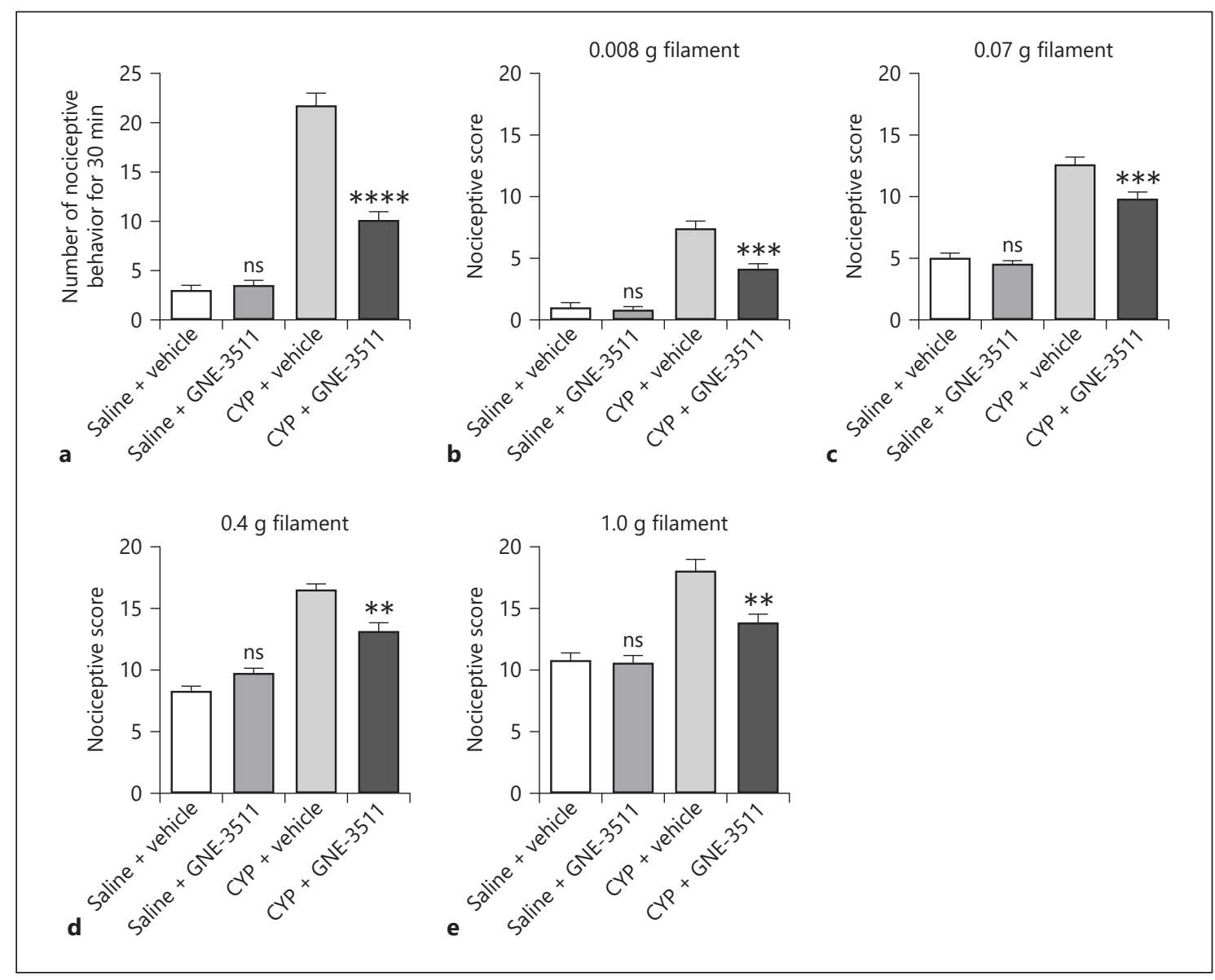

Fig. 6. Inhibition of DLK attenuates pain-like nociceptive behavior caused by CYP. DLK inhibitor GNE-3511 was administered $2 \mathrm{~h}$ prior to CYP treatment (a-e). Nociceptive behavior was observed 3.5-4 h after CYP treatment (a). Hyperalgesia under different von Frey hairs, 0.008 (b), $0.07(\mathbf{c}), 0.4(\mathbf{d})$, and $1.0 \mathrm{~g}(\mathbf{e})$, was evaluated
$4 \mathrm{~h}$ after CYP treatment. $n=10$. Significance was evaluated between vehicle and GNE-3511 groups in the same conditions. ${ }^{* *} p<$ $0.01,{ }^{* * *} p<0.001$, and ${ }^{* * * *} p<0.0001$. Error bars represent SEM. ns, not significant; DLK, dual leucine zipper kinase; CYP, cyclophosphamide. treated mice (Fig. 4a) and deletion of neuronal DLK prevented this CYP-induced MPO activation, while vehicle treatment in $\mathrm{cKO}$ mice had no impact on MPO activity.

We then measured cytokine and chemokine levels in bladder tissue. Consistently, we found that CYP treatment increased MCP-1 (Fig. 4b), TNF-a (Fig. 4c), and IL-1ß (Fig. 4d) levels in WT mice. Vehicle treatment had no impact on bladder inflammation in mice with conditional deletion of DLK in sensory neurons. Importantly, these cytokine and chemokine levels were significantly lowered in CYP-treated cKO mice compared to those in CYP-treated WT mice, indicating that conditional DLK deletion prevented CYP-induced bladder inflammation.

Neuronal Dual Leucine Zipper Kinase Mediates Inflammation

\section{DLK Mediates CYP-Induced Activation of c-Jun in Dorsal Root Ganglia}

We further investigated potential intracellular signaling regulated by DLK during CYP-induced pain and inflammation. We hypothesized that c-Jun is involved in loss of neuronal-DLK-mediated suppression of CYP-induced pain and inflammation. To test this hypothesis, we examined the level of c-Jun phosphorylation in the DRGs of WT and cKO mice treated with vehicle or CYP. Western blot showed a strong induction of $\mathrm{p}$-c-Jun in the DRGs of WT mice following CYP treatment, which was absent in cKO mice treated with CYP (Fig. 5a). To confirm this finding, we stained DRG sections with p-c-Jun and Tuj1 antibodies to label neuronal cells expressing $\mathrm{p}$ c-Jun (Fig. 5b). We found that, consistently, CYP treat- 


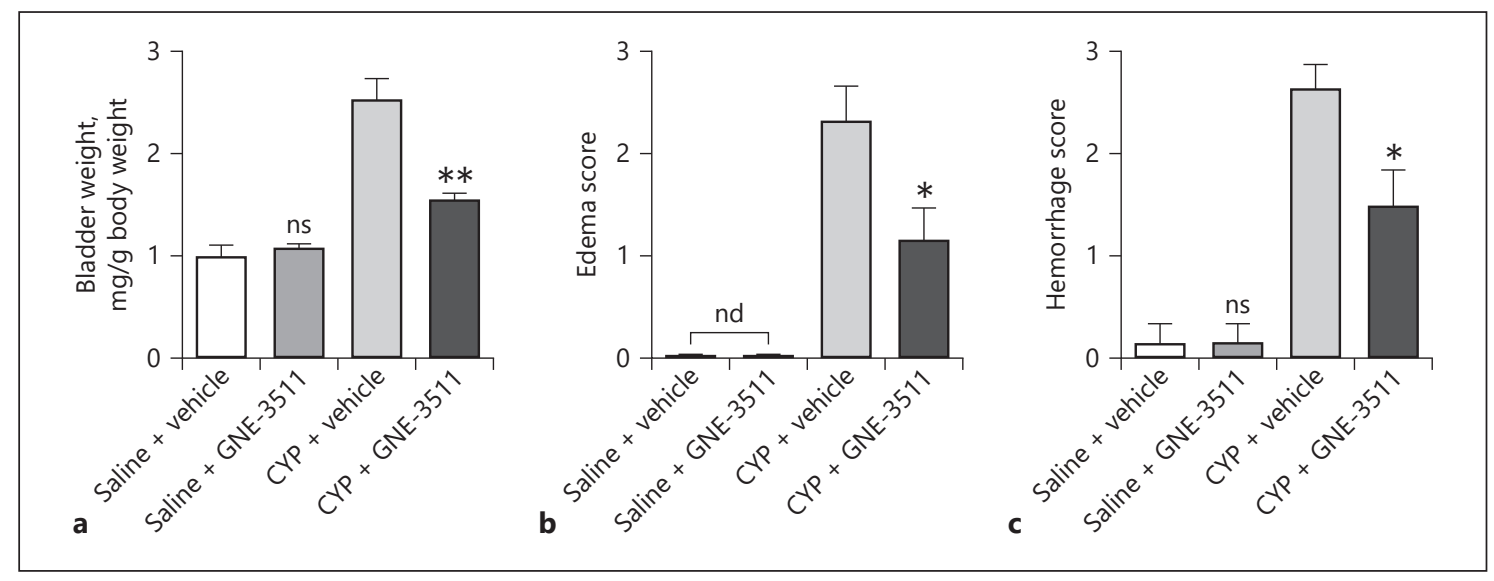

Fig. 7. Inhibition of DLK decreases edema and hemorrhage caused by CYP. Bladder tissues from vehicle- and GNE-3511-treated mice were harvested after cystitis induction $(\mathbf{a}-\mathbf{c})$. Also, wet weight of empty bladder (a), macroscopic edema (b), and hemorrhage (c) were evaluated. $n=6$. Significance was evaluated between vehicle and GNE-3511 groups in the same conditions. ${ }^{*} p<0.05$ and ${ }^{* *} p<$ 0.01 . Error bars represent SEM. ns, not significant; DLK, dual leucine zipper kinase; CYP, cyclophosphamide. ment elicited a drastic increase in the ratio of p-c-Junpositive neurons which was absent in mice deficient in neuronal DLK (Fig. 5c).

\section{DLK Inhibition Suppressed CYP-Induced Pain-Like \\ Nociceptive Behavior in Mice}

To confirm the role of DLK in CYP-induced cystitis in mice, we inhibited DLK with a DLK inhibitor GNE-3511 and assessed CYP-induced pain-like nociceptive behavior. We found that GNE-3511 treatment alone had no significant impact on the number of nociceptive behavior (Fig. 6a) or nociceptive score during the von Frey assay (Fig. 6b-e). Importantly, administration of GNE-3511 in CYP-treated mice significantly reduced the number of nociceptive behavior as well as nociceptive score, suggesting that inhibition of DLK by GNE-3511 suppressed CYP-induced nociceptive behavior in mice.

\section{DLK Inhibition Suppressed CYP-Induced Edema and Hemorrhage in Mouse Bladder}

Finally, we assessed the effects of DLK inhibition on CYP-induced bladder edema and hemorrhage. Consistent with DLK deficiency in sensory neurons, inhibition of DLK by GNE-3511 had no impact on bladder weight (Fig. 7a) and did not induce bladder edema (Fig. 7b) or hemorrhage (Fig. 7c). On the other hand, CYP-induced increase in bladder weight, bladder edema, and hemorrhage was partially and significantly suppressed by GNE3511 , suggesting that pharmacological inhibition of DLK alleviated CYP-induced bladder pathology.

\section{Discussion}

In this study, using a mouse model of CYP-induced cystitis, we have explored the role of DLK in the pathogenesis of bladder pain. We show here that genetic ablation of DLK in sensory neurons or pharmacological inhibition of DLK suppressed pain-like nociceptive behavior and alleviated bladder pathology in mice treated with CYP. Deletion of neuronal DLK reduced mast cells in bladder tissue, suppressed histamine release to the urine, inhibited neuronal activation in the spinal cord, and suppressed inflammatory responses. Our findings in the involvement of DLK in CYP-induced cystitis were also confirmed by pharmacological inhibition of DLK. We showed that c-Jun signaling in DRG might be involved in the pathogenesis of CYP-induced cystitis. Our results, thus, for the first time reveal a critical role of the DLK signaling in sensory neurons in mediating the pathogenic process of cystitis induced by CYP treatment. Our findings with both pharmacological inhibition and genetic ablation of DLK demonstrate that DLK signaling at least partially contributes to the inflammatory responses and nociceptive behavior in mice with cystitis.

Consistent with the neurogenic inflammation and neuropathic pain caused by cystitis, it is notable that ablation of DLK in sensory neurons is sufficient to alleviate bladder pain and inflammation. This is in line with our observation of reduced c-Fos expression and hence reduced neuron activation in the spinal cord. c-Fos expression has long been regarded as a marker for activated spi- 
nal nociceptive neurons in pain models [18]. Nociceptor sensory neurons are an important mechanism in the protection of organisms, and they elicit pain in order to prevent danger [19]. Bladder pain in patients with interstitial cystitis is associated with neuropathic pain [20]. Previous studies have shown that several proteins expressed in sensory neurons such as transient receptor potential ankyrin-1 and Adral play important roles in bladder pain [21], suggesting that sensory neurons may be involved in bladder pain associated with cystitis. Consistent with this possibility, our study showed that specific deletion of DLK in sensory neurons was sufficient to alleviate CYPinduced bladder pathology.

While the exact cause of cystitis is not clear, several pathophysiology mechanisms have been proposed and mast cell activation is one potential mechanism. Increased mast cell count has been detected in conditions with overactive bladder including interstitial cystitis [22]. The chemokine MCP-1, which is elevated in the bladder tissue and urine samples of patients with interstitial cystitis, is important for the degranulation and hence activation of mast cells [23]. In a rat model of cystitis induced by protamine sulfate and lipopolysaccharide, along with mast cell activation, MCP-1 is upregulated which is accompanied with and important for increased release of histamine from mast cells [24]. Consistently, in our mouse model of cystitis induced by CYP, we also detected a significant increase in mast cell counts as well as increased levels of MCP-1 in bladder tissue and histamine in urine, which were suppressed by DLK deletion in sensory neurons. Mast cells are a critical component of the immune system, playing important roles in inflammatory responses [25]. Along with the changes in mast cell counts in patients with cystitis and animal models of cystitis, we also observed a drastic upregulation of proinflammatory cytokines including TNF- $\alpha$ and IL-1ß as well as neutrophil accumulation in the bladder tissue in response to CYP administration in mice. Our important finding that depletion of neuronal DLK alleviated CYP-induced inflammation suggests that DLK may be involved in neurogenic inflammation in cystitis and that inhibition of DLK may be an effective therapeutic strategy for cystitis.

In an effort to explore the intracellular signaling downstream of DLK in mediating the effect of DLK depletion in CYP-treated mice, we assessed c-Jun activation. Previously, it has been shown that deletion of DLK in sensory neurons by Wntla-Cre-mediated recombination lead to reduced c-Jun phosphorylation and hence reduced c-Jun activation following sciatic nerve injury [13]. Similarly, we also observed suppression of c-Jun activation induced by DLK deletion in mice with cystitis. Although beyond the scope of our current study, it is of great interest to understand the more detailed intracellular molecular mechanism underlying DLK-mediated pain regulation in cystitis. This will be one focus of our future study, and we will also explore how modulation of other signaling components downstream of DLK will impact bladder pain associated with cystitis. Doing so may promote the identification of more therapeutic targets for the treatment of bladder pain associated with cystitis. Nevertheless, our finding that depletion of neuronal DLK alleviated nociceptive behavior together with the previous finding that DLK deletion blocks development of neuropathic pain induced by sciatic nerve injury suggests that suppressing DLK may be an effective therapeutic strategy for treatment of neuropathic pain in patients with cystitis or other nerve injuries [11]. In fact, we found that administration of a pharmacological inhibitor of DLK was sufficient to alleviate CYP-induced pain-like nociceptive behavior and bladder pathology including edema and hemorrhage, confirming the potential of DLK inhibition as a therapeutic strategy. Consistently, previous studies also suggest involvement of DLK in neuronal degeneration and a neuroprotective role of DLK deletion in neurodegenerative diseases $[12,26]$.

\section{Conclusion}

In summary, we for the first time identified a connection between DLK in sensory neurons and bladder pain associated with CYP-induced cystitis. Our finding that DLK inhibition not only alleviates bladder pain but also suppresses neurogenic inflammation warrants further investigation of DLK inhibition as an intervention for cystitis and other neuropathic pain disorders.

\section{Statement of Ethics}

Animal studies were approved by the ethics committee of Renji Hospital, School of Medicine, Shanghai Jiao Tong University.

\section{Conflict of Interest Statement}

The authors declare no conflicts of interest. 


\section{Funding Sources}

This work was supported by the Shanghai Integrated Traditional Chinese and Western Medicine Project (zhyyzxyjxzx-2016016).

\section{References}

1 Patnaik SS, Laganà AS, Vitale SG, Butticè S, Noventa M, Gizzo S, et al. Etiology, pathophysiology and biomarkers of interstitial cystitis/painful bladder syndrome. Arch Gynecol Obstet. 2017;295(6):1341-59.

2 Sant GR, Kempuraj D, Marchand JE, Theoharides TC. The mast cell in interstitial cystitis: role in pathophysiology and pathogenesis. Urology. 2007;69(4 Suppl 1):34-40.

3 Nazif O, Teichman JM, Gebhart GF. Neural upregulation in interstitial cystitis. Urology. 2007;69(4 Suppl 1):24-33.

4 Rudick CN, Bryce PJ, Guichelaar LA, Berry RE, Klumpp DJ. Mast cell-derived histamine mediates cystitis pain. PLoS One. 2008;3(5):e2096.

5 Theoharides TC, Alysandratos KD, Angelidou A, Delivanis DA, Sismanopoulos N, Zhang B, et al. Mast cells and inflammation. Biochim Biophys Acta. 2012;1822(1):21-33.

6 Gupta K, Harvima IT. Mast cell-neural interactions contribute to pain and itch. Immunol Rev. 2018;282(1):168-87.

7 Kaur G, Singh N, Jaggi AS. Mast cells in neuropathic pain: an increasing spectrum of their involvement in pathophysiology. Rev Neurosci. 2017;28(7):759-66.

8 Leiby BE, Landis JR, Propert KJ, Tomaszewski JE; Interstitial Cystitis Data Base Study G. Discovery of morphological subgroups that correlate with severity of symptoms in interstitial cystitis: a proposed biopsy classification system. J Urol. 2007;177(1):142-8.

9 Whitmore KE, Theoharides TC. When to suspect interstitial cystitis. J Fam Pract. 2011; 60(6):340-8.

10 Martins JP, Silva RB, Coutinho-Silva R, Takiya CM, Battastini AM, Morrone FB, et al. The role of $\mathrm{p} 2 \mathrm{x} 7$ purinergic receptors in inflammatory and nociceptive changes accom-

\section{Author Contributions}

Chen Jiang, Weilin Fang, Tingting Lv, and Yinjun Gu did the experiments and collected and analyzed the data. Chen Jiang, Weilin Fang, and Jianwei Lv wrote the manuscript. Jianwei Lv conceived the study and coordinated the research. panying cyclophosphamide-induced haemorrhagic cystitis in mice. Br J Pharmacol. 2012;165(1):183-96.

11 Wlaschin JJ, Gluski JM, Nguyen E, Silberberg $\mathrm{H}$, Thompson JH, Chesler AT, et al. Dual leucine zipper kinase is required for mechanical allodynia and microgliosis after nerve injury. Elife. 2018;7:e33910.

12 Le Pichon CE, Meilandt WJ, Dominguez S, Solanoy H, Lin H, Ngu H, et al. Loss of dual leucine zipper kinase signaling is protective in animal models of neurodegenerative disease. Sci Transl Med. 2017;9(403):eaag0394.

$13 \mathrm{Hu}$ Z, Deng N, Liu K, Zeng W. Dlk mediates the neuronal intrinsic immune response and regulates glial reaction and neuropathic pain. Exp Neurol. 2019;322:113056.

14 Patel S, Cohen F, Dean BJ, De La Torre K, Deshmukh G, Estrada AA, et al. Discovery of dual leucine zipper kinase (dlk, map3k12) inhibitors with activity in neurodegeneration models. J Med Chem. 2015;58(1):401-18.

15 Russo A, DiAntonio A. Wnd/dlk is a critical target of fmrp responsible for neurodevelopmental and behavior defects in the drosophila model of fragile x syndrome. Cell Rep. 2019; 28(10):2581-e5.

16 Zurborg S, Piszczek A, Martínez C, Hublitz P, Al Banchaabouchi M, Moreira P, et al. Generation and characterization of an advillin-cre driver mouse line. Mol Pain. 2011;7:66.

17 Matsunami M, Miki T, Nishiura K, Hayashi $\mathrm{Y}$, Okawa Y, Nishikawa $\mathrm{H}$, et al. Involvement of the endogenous hydrogen sulfide/ca(v) 3.2 t-type ca2+ channel pathway in cystitis-related bladder pain in mice. Br J Pharmacol. 2012; 167(4):917-28.

18 Hossaini M, Duraku LS, Kohli SK, Jongen JL, Holstege JC. Spinal distribution of c-fos acti- vated neurons expressing enkephalin in acute and chronic pain models. Brain Res. 2014; 1543:83-92.

19 Pinho-Ribeiro FA, Verri WA Jr, Chiu IM. Nociceptor sensory neuron-immune interactions in pain and inflammation. Trends Immunol. 2017;38(1):5-19.

20 Cory L, Harvie HS, Northington G, Malykhina A, Whitmore K, Arya L. Association of neuropathic pain with bladder, bowel and catastrophizing symptoms in women with bladder pain syndrome. J Urol. 2012;187(2):5037.

21 DeBerry JJ, Schwartz ES, Davis BM. Trpa1 mediates bladder hyperalgesia in a mouse model of cystitis. Pain. 2014;155(7):1280-7.

22 Gamper M, Regauer S, Welter J, Eberhard J, Viereck V. Are mast cells still good biomarkers for bladder pain syndrome/interstitial cystitis? J Urol. 2015;193(6):1994-2000.

23 Castellani ML, De Lutiis MA, Toniato E, Conti F, Felaco P, Fulcheri M, et al. Impact of rantes, mcp-1 and il-8 in mast cells. J Biol Regul Homeost Agents. 2010;24(1):1-6.

24 Lv J, Huang Y, Zhu S, Yang G, Zhang Y, Leng $J$, et al. Mcp-1-induced histamine release from mast cells is associated with development of interstitial cystitis/bladder pain syndrome in rat models. Mediators Inflamm. 2012;2012:358184.

25 Gonzalez-de-Olano D, Alvarez-Twose I. Mast cells as key players in allergy and inflammation. J Investig Allergol Clin Immunol. 2018; 28:365-78.

26 Larhammar M, Huntwork-Rodriguez S, Jiang Z, Solanoy H, Sengupta Ghosh A, Wang B, et al. Dual leucine zipper kinase-dependent perk activation contributes to neuronal degeneration following insult. Elife. 2017;6:e20725. 